PR-13

\title{
NANOENCAPSULATION OF PESTICIDES: SUSTAINABLE PERSPECTIVE IN AGRICULTURE
}

\author{
V. Madhavi, ${ }^{1 *}$ A. Vijaya Bhaskar Reddy, ${ }^{2}$ G. Madhavi, ${ }^{3}$ N. Bakthavatchala Reddy ${ }^{4}$ \\ ${ }^{1}$ Associate Prof. in Chemistry, \\ BVRIT Hyderabad College of Engineering for Women, Hyderabad. \\ ${ }^{2}$ Centre of Research in Ionic Liquids, Universiti Teknologi PETRONAS, \\ Seri Iskandar 32610, Perak, Malaysia. \\ ${ }^{3}$ Department of Chemistry, Sri Venkateswara University, \\ Tirupati 517502, India. \\ ${ }^{4}$ Ural Federal University, Chemical Engineering Institute Yekaterinburg, \\ 620002, Russian Fedaration. \\ *Corresponding author, E-mail: madhuchem9@gmail.com
}

\begin{abstract}
The concern towards the increasing challenges of sustainable agriculture made the researchers towards significant technological development and innovations in recent years. In particular Nanotechnology offers potential solutions to improve agricultural production assuring sustainability. The incorporation of nanotechnology as a means of nanopesticides delivers the pesticides appropriately i.e., targeted delivery of pesticides by lowering the indiscriminate use of conventional pesticides. The assets of a nanoencapsulated pesticide formulation are controlled releasing properties with enhanced solubility, specificity, permeability and stability. They provide in-built switches to control the release and subsequent availability of pesticides to be used effectively over a given period of time and their design enables them to resist the severe environmental processes such as leaching, evaporation, photolytic hydrolytic and microbial degradation over conventional pesticides. This review provides fundamental information to critically assess the significant role of nanotechnology for encapsulation of pesticides.
\end{abstract}

